\title{
Blood ethanol testing
}

\author{
NORMAN B. COFFMAN, PhD \\ JOHN J. FERNANDES, DO
}

The justification for blood ethanol testing springs from the ubiquitous abuse of alcoholic beverages. Those dealing with patients who abuse alcohol must understand the correlations between dose, time, blood level, and behavior. Enumerated here are the strengths of various testing methods and the requisites for legally defensible testing. The challenge remains to determine blood ethanol levels in such a manner that the results are scientifically valid and legally defensible.

(Key words: Blood ethanol testing, alcohol abuse, laboratory medicine, alcohol intoxication, forensics)

Take your hat and a bottle of good whiskey. Throw your hat on the bedpost. Drink the whiskey until you see two hats. Get into bed and stay there.

-Old English cure for head colds

Prohibition failed. So, in 1936, the American Medical Association (AMA) set up a committee to examine the effects of drinking on motor vehicle accidents. This same AMA committee also looked at the medical and legal aspects of measurements of ethanol in biologic fluids. Subsequently, Indiana became the first state to enact a law defining intoxication in terms of blood alcohol content (BAC). (Note:

From the Department of Pathology and Laboratory Medicine, Philadelphia College of Osteopathic Medicine, Philadelphia, $\mathrm{Pa}$, where Dr Coffman is associate professor of pathology and laboratory medicine. Dr Fernandes is currently chairman, Department of Pathology, Chicago College of Osteopathic Medicine, Downers Grove, Ill.

Reprint requests to Norman B. Coffman, PhD, Department of Pathology and Laboratory Medicine, Philadelphia College of Osteopathic Medicine, 4150 City Ave, Philadelphia, PA 19131-1696.
$\mathrm{BAC}$ refers to the ethanol content of whole blood.) The first breath tester for ethanol was introduced in 1954.

In spite of the continuing effort over the years to discourage driving while intoxicated (DWI), more than half a million people are injured annually in the United States in automobile accidents in which ethanol is a factor. Unfortunately, most statistics are kept relative to the prima facie intoxication level of $0.1 \%$ ethanol. It has been the authors' personal observation that many persons involved in alcohol-related accidents have blood ethanol levels below the prima facie level of $0.1 \%$ but greater than the approximately $0.05 \%$ level at which incapacitation is known to begin. It is probably true that our statistics seriously underestimate the real magnitude of the antisocial effects of ethanol. As a matter of fact, in 1989 the then Surgeon General C. Everett Koop recommended that the BAC limit be lowered to $0.08 \%$ immediately and then further lowered to $0.04 \%$ by the year 2000 .

The magnitude of the effects of ethanol abuse on each individual in our society has led to many efforts to provide use of and promote understanding about ethanol testing.

\section{Central nervous system effects of ethanol} The first and the most acute effects of alcohol are on the central nervous system (CNS). It has been found that alcohol first depresses the postsynaptic structures of the reticular activating system and certain sites of the cerebral cortex. Because these regions of the brain usually inhibit excessive, extranormal behavior, the first CNS effects of ethanol appear to be stimulatory. Skills acquired by training are 


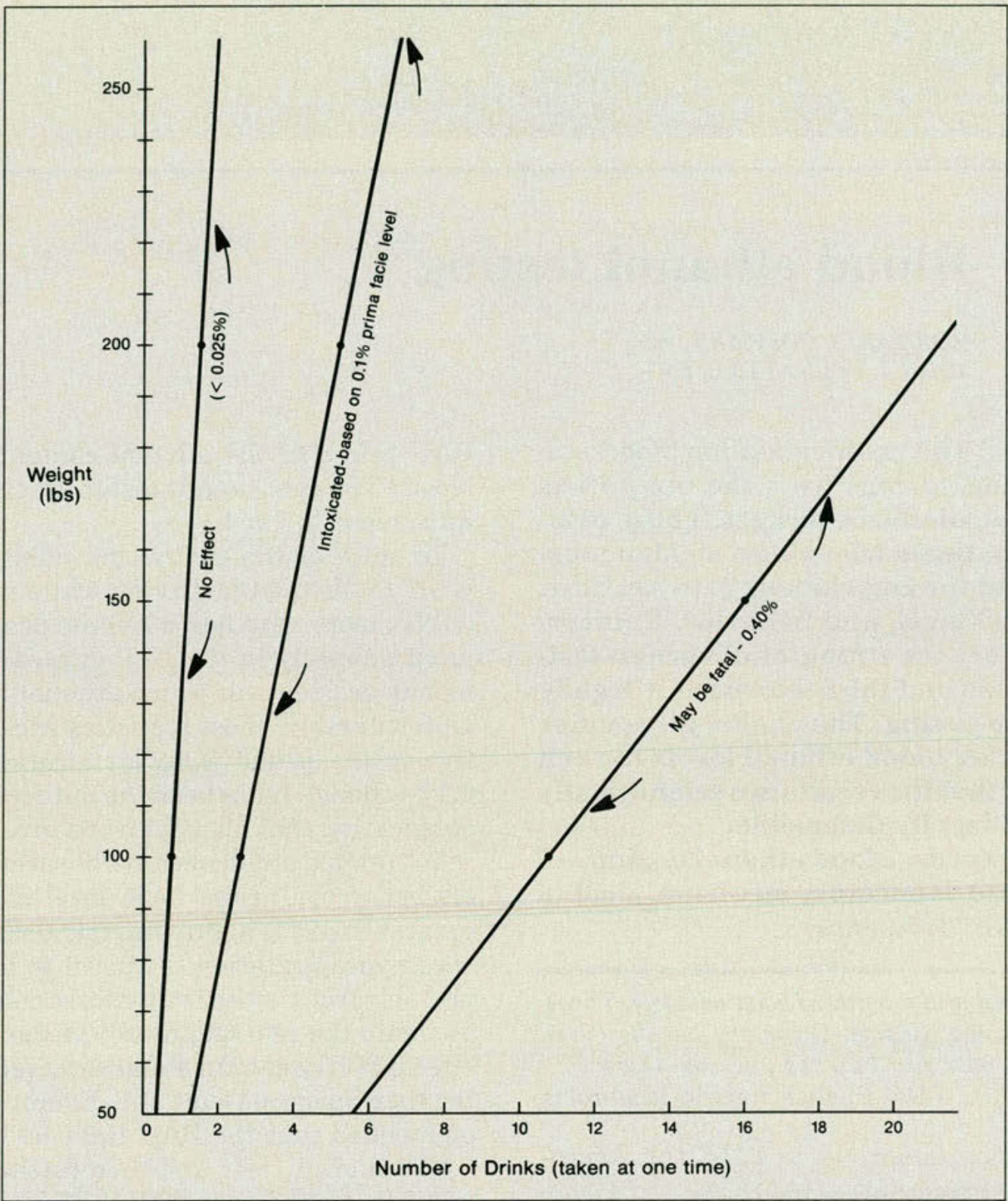

Figure 1. Relationship between drinking and intoxication.

lost soon thereafter, while personal behavior becomes more and more expansive. Later, spinal reflexes are lost, and eventually a state of general anesthesia predominates. At BAC levels above about $0.4 \%$, a dangerous, or even lethal, depression of respiration may occur.

\section{Meaning of blood alcohol levels}

At least two types of correlations are relevant to blood ethanol levels. The first correlation relates consumption to the degree of intoxication. This is the datum of crucial interest to the consumer of alcoholic beverages and to those he or she meets on the highway. The second correlation deals with the relationship between measured BAC levels and degree of intoxication. It is the latter correlation that is usually considered in diagnostic workups.

In looking at the former correlation, one must consider the relationships involving the amount consumed, weight of the individual consuming the alcohol, and time. Once ethanol is absorbed, it rapidly equilibrates with total body water. For this reason, a good relationship exists between consumption, weight, and BAC. As an example, a 70-kg man who drinks 


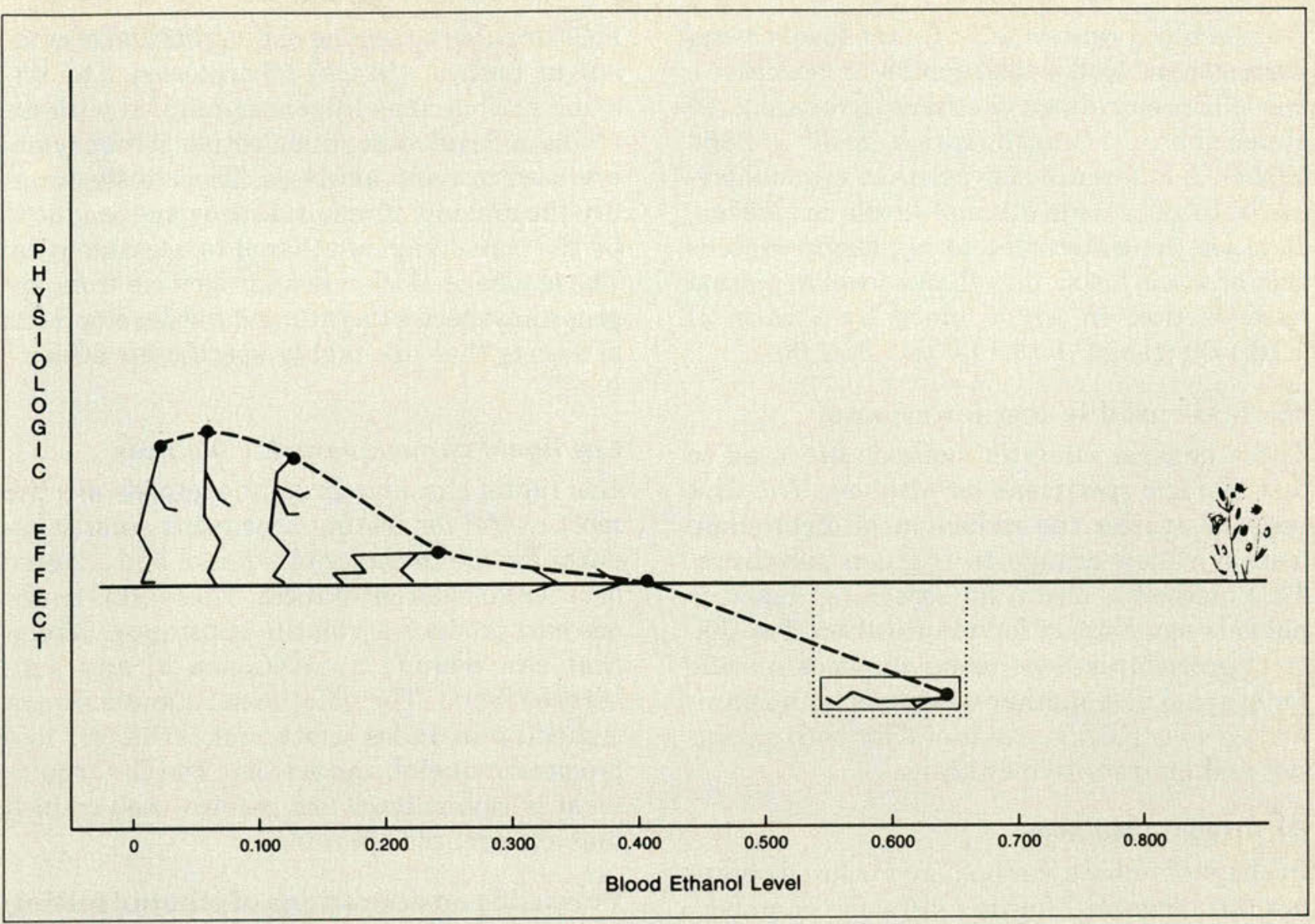

Figure 2. Physiologic effects of blood ethanol levels.

3 ounces of pure ethanol (that is, about three fourths of a glass of straight 100-proof vodka) all at once will achieve a $\mathrm{BAC}$ in the range of $0.09 \%$ to $0.15 \%$. He may or may not still be legally sober. The same amount of ethanol will be derived from 12 ounces of fortified wine or eight 12-ounce cans of beer.

It is quite interesting that our society has chosen to package and serve alcoholic beverages in amounts that will maintain a constant level of intoxication (be metabolized) in the "average" 70 -kg man for a period of about 1 hourthat is, 1 ounce of 100-proof hard liquor, 3 ounces of wine, or 12 ounces of beer. Figure 1 provides a more extensive outline of consumption versus intoxication level.

The second type of correlation is that which exists between measured BAC and degree of inebriation. It is commonly observed that persons who chronically use alcohol-or other drugs - develop the ability to apparently compensate for its effects on their behavior. Yet it is usually found that functions such as reaction time, fine motor control, and critical thinking start to become diminished at a BAC of $0.02 \%$ to $0.03 \%$. At a level of $0.15 \%$, most persons are obviously drunk. Average levels of $0.74 \%$ (range, $0.42 \%$ to $1.77 \%$ ) were found in one study ${ }^{1}$ of acute, fatal intoxication.

Diagnosis of intoxication can be difficult. Care must be taken to exclude diabetic coma, intoxication from other drugs, cardiovascular accidents, and fractures of the skull, all of which may appear with symptoms similar to those of alcohol intoxication. As manufacturers of certain alcoholic beverages (for example, vodka) have well advised us, breath odor is a notoriously unreliable guide for the detection of ethanol ingestion. Figure 2 provides a guide to the relationship between $\mathrm{BAC}$ and inebriation.

Efforts have been made to correlate breath alcohol levels and urine alcohol levels to blood ethanol levels. During the elimination phase, 
a urine/blood ratio of 1.3:1 (urine levels being higher than blood levels) applies in most cases. Blood concentrations of ethanol average 2180 times those in breath (range, 1837 to 2863 times). A difference also exists in ethanol levels in blood versus ethanol levels in plasma. Because the water content of plasma exceeds that of whole blood, the ethanol level in plasma exceeds that in whole blood by a ratio of $1.18: 1.00$ (range, $1.10: 1.00$ to $1.35: 1.00$ ).

\section{Methods used to test for ethanol}

Three general analytic methods are used to test biologic specimens for alcohols. The first method utilizes the reduction of dichromate from a yellow-orange to a green substance. This method is useful for screening, but it is not very satisfactory for quantitation. Two distinct procedures - one using enzymes specific for ethanol and another using gas liquid chromatography (GLC) - are useful for both screening and quantitative analysis.

\section{Dichromate method}

In the dichromate method, an enclosed chamber (for example, Conway diffusion chamber) containing separate compartments for the biologic fluid sample and the reagent is used. The test is set up, and then time is allowed for volatile substances to diffuse from the sample to the reagent. Any reducing substances that reach the reagent will cause the reagent to change from yellow-orange to green. The extent of the color change is an indication of the amount of alcohol in the sample. Alcohols other than ethanol aldehydes, and chloral hydrate are examples of reducing substances that can interfere with this method by also causing the reagent to change from yellow-orange to green.

\section{Enzyme-mediated analytic methods}

Enzyme-mediated analytic methods are widely used. Reagent/instrument packages are manufactured and marketed by several companies (for example, Abbott Laboratories Diagnostics Division, Irving, Tex; Sigma Chemical Co, St Louis, Mo; and the Syva Company, Palo Alto, Calif). These methods have proved to be both accurate and dependable. Usually, they are suit- able for either screening or quantitation of ethanol in routine clinical laboratories. The enzyme alcohol dehydrogenase coupled with nicotinamide adenine dinucleotide, a coenzyme, are used in these analyses. These tests measure the amount of reduced coenzyme produced by the conversion of ethanol to acetaldehyde. The choice of alcohol dehydrogenase from appropriate species has allowed the development of assays that are highly specific for ethanol itself.

\section{Gas liquid chromatographic methods}

Gas liquid chromatographic methods are the most useful for testing specimens simultaneously for the presence of ethanol and a range of other volatile substances. These GLC methods can produce a volatile substances screen that can include quantitation of any substances found. The volatile substances screen typically includes methanol, ethanol, isopropanol, butanol, and acetone. The GLC equipment is sophisticated and requires well-trained and experienced operators.

\section{Forensic considerations of ethanol testing}

As previously mentioned, most states in the United States have laws making it illegal to drive vehicles while under the influence of alcohol. In general, these laws state that persons with blood ethanol levels in the range of $0.05 \%$ to $0.10 \%$ may be intoxicated and that the courts may consider the BAC as evidence together with other evidence to establish intoxication. These laws in general also state that a BAC higher than $0.10 \%$ is in itself (that is, prima facie) evidence of intoxication.

It is important to note that these laws do not state that driving with a BAC of more than $0.05 \%$ is legal. They merely say that other auxiliary evidence is needed for a DWI conviction when the BAC range is $0.05 \%$ to $0.10 \%$. Because of the existence of these DWI laws, much of the blood ethanol testing that is done must be carried out in a manner that is in compliance with forensic requirements and constraints.

The chief requirement for forensically defensible testing is that a chain of evidence be established. This requirement means that some- 
one must be able to go into court and be able to prove by sworn testimony, beyond a reasonable doubt, as to the exact whereabouts of the specimen and as to who had access to and custody of the specimen-beginning from the time the specimen was first obtained up to and including the time the specimen was tested. It must be shown that no opportunity existed at any time for the specimen to be either misidentified or altered or adulterated. The best proof is a complete chain-of-custody document that lists all the required data. Many examples of acceptable chain-of-custody documents are available. One source is Cravey and Baselt. ${ }^{2}$

Data related to the analysis itself must also be available for court presentation. Items related to the analysis include instrument calibration data, quality control data, evidence of review of the test results, and the identity of the analyst. The recordkeeping involved is not difficult. However, the documentation is lengthy, it is time-consuming, and it must be done with exactness.

\section{Summary}

The ubiquitous use-and concomitant abuseof ethanol makes the sequelae of ethanol ingestion a major factor in the practice of medicine. Blood ethanol begins to have an impact on the central nervous system at a much lower level than is generally recognized by either the legal profession or the general public. Most per- sons begin to lose functioning when the blood ethanol level is about $0.04 \%$ to $0.05 \%$, a level much lower than the usual prima facie level of $0.1 \%$.

Several methods are available for the analysis of ethanol in blood. Those most commonly used for ethanol screening are enzyme-based, and several manufacturers produce complete analytic systems. Gas liquid chromatography serves as the best tool for a general volatile substances screen. Forensic considerations often must be recognized in the testing of specimens for ethanol. Although the maintenance of a chain of custody is not difficult, it is timeconsuming and must be carried out with exactness.

1. Baselt RC, Cravey RH: Deposition of Toxic Drugs and Chemicals in Man, ed 3. Chicago, Ill, Year Book Medical Publishers Inc, 1989, pp 322-326.

2. Cravey, RH, Baselt RC (eds): Introduction to Forensic Toxicology. Davis, Calif, Biomedical Publications, 1981, pp 136-137, 260-264.

Gilman AG, Goodman LS, Rall TW, et al (eds): Goodman and Gilman's the Pharmacological Basis of Therapeutics, ed 7. New York, NY, Macmillan Publishing Co, 1985, pp 372.

Klassen CD, Amdur MO, Doull J (eds): Casarett and Doull's Toxicology, the Basic Science of Poisons, ed 3. New York, NY, Macmillan Publishing Co, 1986, pp 648-652.

Porro J: Drunk driving 1980 to 1990: A sobering look. Syva Monitor 1990;8(4):1-6.

Tietz NW (ed): Textbook of Clinical Chemistry. Philadelphia, $\mathrm{Pa}$, WB Saunders Co, 1986, pp 1692-1694; 1703-1706. 